\title{
High-Resolution Coudé-Echelle Spectrometer for the 1.5-m Kazan University Telescope at the Turkish National Observatory
}

\section{FAIG MUSAEV ${ }^{1}$ and ILFAN BIKMAEV ${ }^{2}$}

1 Special Astrophysical Observatory, Russian Academy of Sciences Nizhnij Arkhyz, Russia 357147

2 Engelhardt Astronomical Observatory, Kazan State University Kazan, Russia 420008

Modern tasks of stellar spectroscopy demand the registration of spectra with high spectral resolution and high signal-to-noise ratio over a broad spectral region. The only way to realize these requirements simultaneously is with an echelle spectrometer working with a large-format CCD. For 1-3-m class telescopes such spectrometers would be installed in separate coudé rooms. We propose a project of modern coudé instrumentation for the 1.5-m Kazan University telescope being installed at the Turkish National Observatory in 1997. The use of two echelle gratings (R2 and R4) with prisms as cross-dispersers and two optical cameras will provide a spectral resolution up to $R=200,000$ in a wide spectral range from 3500 to $9500 \AA$. As a basic model, the optical scheme of the coudé-echelle spectrometer of the 1-m SAO RAS telescope has been adopted. This spectrometer was designed and constructed during 199395 and is now providing high-quality spectral data (Musaev 1996, Astronomy Letters, v. 22, n. 10).

To obtain high-quality spectra during long observing periods, there are some important technical requirements for the telescope tower: $(a)$ the coudé room should be well temperature-controlled and mechanically stabilized, $(b)$ there should be no (or minimal) heat sources inside the tower, and (c) the telescope's automatic control system should be situated in a separate room at the 1st floor, or even outside the tower. The telescope tower is now being constructed by our Turkish collaborators according to these requirements (http://astroa.physics.metu.edu.tr).

Some of the spectrometer's optical elements have been manufactured in Russia during 1995-96 and we expect that observations will start in 1998. The main scientific goals for the coudé instrumentation of this $1.5-\mathrm{m}$ telescope are: (a) detailed chemical composition determination of the atmospheres of hot and cool stars down to 8th magnitude, $(b)$ study of spectral line profiles and possible time variations of line profiles with a resolution $R=40,000-200,000$ and $\mathrm{S} / \mathrm{N}$ ratio $>100,(c)$ the production of stellar atlases of a sample of bright stars of different spectral types, $(d)$ a search for low-amplitude (50-500 $\mathrm{m} \mathrm{s}^{-1}$ ) variations of radial velocities of cool stars, $(e)$ study of the spectra of magnetic A-F stars, and (f) study of the local interstellar medium. 